\title{
Inklusiver Sachunterricht zwischen Kind- und Materialorientierung - Mediennutzung und Motive der Medienauswahl im Fokus einer explorativen Lehrkräftebefragung
}

\author{
René Schroeder
}

Online publiziert: 17. Januar 2020

(C) Der/die Autor(en) 2020

Zusammenfassung Der vorliegende Beitrag referiert empirische Befunde einer zweiphasigen Lehrkräftebefragung zur Sachunterrichtpraxis in Nordrhein-Westfalen. Der Fokus der dargestellten Ergebnisse liegt hierbei auf der Mediennutzung sowie den damit verbundenen Motiven zur Medienauswahl der Lehrkräfte im inklusiven Sachunterricht. Die vorliegenden Daten wurden im Rahmen eines integrierten Mixed-Methods-Designs, bestehend aus einer teilstandardisierten Fragebogenerhebung $(n=80)$ sowie vertiefenden Experteninterviews $(n=10)$ mit einer Teilstichprobe der zuvor befragten Sachunterrichtslehrkräfte, gewonnen. Ergebnisse aus der statistischen wie auch qualitativ-inhaltsanalytischen Auswertung der quantitativen und qualitativen Daten werden mit Schwerpunkt auf die Mediennutzung sowie möglichen Einflussfaktoren hierauf analysiert und interpretiert. Dies geschieht vor dem Hintergrund der besonderen Bedeutung, die einem vielfältigen und differenzierten Medien- und Materialangebote für ein Kind orientiertes Lernen aus Perspektive einer inklusiven Sachunterrichtdidaktik zugeschrieben wird. Ebenso wird reflektiert, inwieweit sich eine aus bereits vorliegenden Studien erkennbare Materialorientierung von Sachunterrichtlehrkräften, bei der didaktisch-methodische Entscheidungen durch verfügbare Medien und Materialien determiniert werden, auch in den eigenen Befunden widerspiegelt. Im Ausblick werden mögliche Schlussfolgerungen für einen inklusiven Sachunterricht hinsichtlich zukünftiger fachdidaktischer Forschung zu barrierefreien Lehr-Lernmaterialien abgeleitet.

Schlüsselwörter Inklusiver Sachunterricht · Lehrkräftebefragung ·

Mediennutzung · Mixed-Methods-Design

R. Schroeder $(\bowtie)$

Fakultät für Erziehungswissenschaft, AG 3: Schultheorie mit dem Schwerpunkt Grund- und

Förderschulen, Universität Bielefeld, Universitätsstraße 25, 33615 Bielefeld, Deutschland

E-Mail: rene.schroeder@uni-bielefeld.de 


\title{
Inclusive education in general studies, child or media centered-an explorative teachers' survey about media use in teaching and teachers' beliefs
}

\begin{abstract}
In this article empirical results from a teacher review about general studies in North-Rhine-Westphalia Germany are reported. The article focuses on the use of media in teaching and teachers' beliefs about practices regarding media in general studies and inclusive education. The study is designed as mixed-methods with a partly standardized questionnaire $(n=80)$ in the first step, succeeded by expert interviews to deepen up knowledge about teachers' practices and beliefs. Data is analyzed by using descriptive statistics as far as quantified data is concerned. The interviews are analyzed by using strategies of quality content analysis. Both types of data are triangulated and interpreted under the topic of media use in teaching and influences on these practices. The interpretation is led by the assumption of a particular importance of multiple means of representation and expression in inclusive education in the field of general studies. On the one hand this focuses on a child-centered perspective on learning. On the other hand, resent research shows the influence of available media and materials on teaching practices. To a certain degree teachers' beliefs and decisions are determined by ready to use teaching aids. Against this background a critical commenting of the reported results is necessary. Concluding the need for future research and development in the field of accessibility and media is emphasized to further inclusive education in general studies.
\end{abstract}

Keywords Inclusive education - General studies · Teacher survey • Use for media · Mixed-methods-design

\section{Unterrichtsmedien in ihrer Bedeutung für den Sachunterricht}

Die Frage nach geeigneten Lernmaterialien und Unterrichtsmedien stellt einen relevanten Planungsaspekt in der Gestaltung gelingenden Sachunterrichts dar (Kahlert 2016, S. 151). Indem sachunterrichtliche Planungskonzeptionen (etwa Tänzer und Lauterbach 2010) vorrangig an die allgemeindidaktische Traditionslinie einer bildungstheoretischen bzw. kritisch-kommunikativen Didaktik (Klafki 2007) anknüpfen, wird jedoch weniger von einer interdependenten Gleichrangigkeit von Zielen, Inhalten, Methoden und Medien im Planungsprozess ausgegangen, sondern diese folgen einem „Primat der Sache“ (Köhnlein 2010). Die Entscheidung über Unterrichtsmedien, die den Schülerinnen und Schülern eine individuell bedeutsame Sachbegegnung ermöglichen, ist demnach gegenüber dem Entscheidungsprozess der Themen- und Zielklärung nachgelagert, muss aber für das konkrete Planungshandeln als wesentlicher Bestandteil erscheinen. So ist die Medien- und Materialauswahl für den Sachunterricht enggebunden an die zu realisierende Verlaufsplanung im Kontext der inhaltlichen und zeitlichen Strukturierung sowie der Methodenauswahl (Kahlert 2016, S. 241). Inhaltlich-methodische wie auch intentionale Entscheidungen determinieren die hierzu notwendigen Unterrichtsmedien, wie auch die Auswahl eines bestimmten Mediums spezifische Lernwege und -ziele vorgeben kann. Diese Ver- 
schränkung gilt umso mehr, wenn es darum geht geöffnete und differenzierte Lernumgebungen im Sachunterricht zu gestalten. So spricht Peschel (2016, S. 9) von einer inklusiven Mediendidaktik für den Sachunterricht, bei der sich das Attribut inklusiv auf die Ideen bezieht, dass ein Lernen mit Medien und das Lernen über Medien in „,die didaktische Konstruktion einer Lehr-Lern-Situation“ (ebd., S. 10) integriert gedacht werden soll. „Der Stellenwert der Medien für das Lernen beruht auf der Annahme, dass Mediennutzung eine Form der Erfahrung darstellt, durch die gelernt werden kann" (Tulodziecki 2011, S. 402). Dies stützt die Prämisse einer relevanten Interdependenz von Themen, Methoden und Medien im Sachunterricht als wechselseitiger Orientierung thematischer, methodischer und medialer Entscheidungsfelder, indem sich einerseits eine Abhängigkeit der medialen Möglichkeiten von den behandelten Themen und eingesetzten Methoden ergibt, und andererseits die genutzten Medien inhaltlich-methodische Realisationsformen des Unterrichts in Teilen präformieren (Heckt 2005, S. 449f.). Aktuelle Planungskonzeptionen für inklusiven Sachunterricht (etwa Gebauer und Simon 2012; Kaiser und Seitz 2017) fußen zwar weiterhin auf einer bildungstheoretischen Didaktik (Klafki 2007), stellen demgegenüber jedoch die Kindorientierung voran, indem inhaltlich-intentionale Entscheidungen Gegenstand eines gemeinsamen Aushandlungsprozesses sein sollen.

Je nach planungstheoretischer Position im Entscheidungsfeld zwischen Sachprimat, Kindorientierung oder Interdependenzverhältnis lässt sich die Rolle bzw. Funktion von Medien und Materialien im Sachunterricht unterschiedlich systematisch fassen (Mitzlaff 2016; Unglaube 2015). Mit Fokus auf deren didaktische Intention, etwa zur Präsentation, Kommunikation oder interaktiven Aneignung von Lerninhalten, können diese von der Perspektive der Lehrkraft als Lehrmittel verstanden, oder als Lernmittel der Schülerinnen und Schüler bestimmt werden (Mitzlaff 2016, S. $23 \mathrm{f}$.). Inwieweit Lernmittel dabei eine offene und selbsttätige Sachbegegnung zulassen, Differenzierung ermöglichen bzw. vorgeben oder stark vorstrukturiert als didaktisierte Arbeitsmittel Lernwege determinieren, ist mit dem Begriff zunächst unbestimmt (Heckt 2005, S. 449). So zeigt sich an vorliegenden Systematisierungsversuchen von Mitzlaff (2016) und Unglaube (2015) bereits in den genutzten Termini nicht nur eine Unterscheidung begrifflicher Art, sondern mit Arbeitsmitteln (Unglaube 2015, S. 443) richtet sich der Blick auf den Arbeits- bzw. Lernprozess der Kinder und inwiefern dieser intentional-inhaltlich oder methodisch stark vorstrukturiert ist (z.B. Experimentierboxen, Modelle, Sachtexte) oder erfahrungsoffen und unbestimmt (z. B. Alltagsmaterialien) bleibt. Mit dem Begriff Lehr- und Lernmedien kann hingegen die didaktische Intention, also der funktionale Aspekt (Mitzlaff 2016, S. 24f.) im Kontext der Unterrichtsplanung eher betont werden, obwohl diese mit dem Begriff der Arbeitsmittel (Unglaube 2015, S. 494f.) nicht gänzlich wegfällt. Lernmedien und Arbeitsmittel könnten in Abgrenzung zu Lehrmedien eher synonym gebraucht werden, obwohl mit dem Arbeitsbegriff der sichtbar Tätigkeitsaspekt hervorgehoben wird, gegenüber Lernen als internem Prozess. Weiterhin ist die didaktische Funktion bei vorstrukturierten Medien ein Merkmal des Materials selbst, wohingegen sie bei unstrukturierten Alltagsmaterialien erst durch damit verbundene Lernaufgaben entsteht oder durch die Kinder selbst diesen in der Sachbegegnung zugeschrieben wird. 
Es wird deutlich, dass der Einsatz von Lehr- und Lernmedien im Sachunterricht enggekoppelt ist an die intentionale wie auch methodisch-organisatorische Ebene des Unterrichts, indem bestimmte Lern- und Aneignungswege durch den Medienund Materialeinsatz ermöglicht, vorgezeichnet oder verhindert werden. Hier ist daher nach ihrer Funktion in einem inklusiven Sachunterricht zu fragen, da mit der Vielfalt der Kinder (Wocken 2013) in einem inklusiven Unterricht auch die Vielfalt der medial vermittelten Verstehens-, Erfahrungs-, Interaktions- und Kommunikationsprozesse zunehmen dürfte.

\section{Medien als vielfältige Zugangsweisen und Repräsentationsformen in einem inklusiven Sachunterricht}

Gemäß dem Fokus der Untersuchung auf inklusiven Sachunterricht ist darzulegen, wie sich die Frage des Medieneinsatzes und damit auch möglicher Entscheidungenkriterien unter theoretisch-konzeptioneller Perspektive einer inklusiven Sachunterrichtsdidaktik (etwa Pech et al. 2018) neu oder anders stellt. Dabei kann mit Kaiser und Seitz (2017, S. 8) inklusiver Sachunterricht als ,,guter individualisierender und kommunikativer Sachunterricht mit besonderem Augenmerk auf den sachbezogenen Austausch der Kinder untereinander" verstanden werden. Die Anerkennung jeden Kindes (Personalität), Mitbestimmung und Zugehörigkeit (Sozialität) sowie eine Anregung über offene und selbstdifferenzierte Aufgaben (Komplexität) werden als Leitprinzipien (ebd., S. 12) formuliert.

Indem ein Lernumgebungskonzept (Schomaker 2013; Seitz 2005) als eine wesentliche Realisationsform inklusiven Sachunterrichts gedacht wird, rückt die Bedeutung eines vielfältigen Medien- und Materialangebots in den besonderen Fokus (fach-)didaktischer Betrachtungen. Ziel ist ein individualisiertes, handlungsorientiertes und selbstorganisiertes Lernen in gemeinsamer Kooperation zum Kern der Sache (Seitz 2008). Die prinzipielle „Gleichrangigkeit verschiedener Interaktions- und Repräsentationsformen sowie Abstraktionsniveaus für alle Lernenden" (Pech et al. 2017, S. 124) wird als wesentliches Element eines inklusiven Sachunterrichts konstitutiv. Die bereitgestellten Medien und Materialien sollen mit dem Ziel ausgewählt werden, allen Kindern vielfältige Sachbegegnungen und somit Lernwege auf verschiedenen Aneignungsebenen, ,basal-perzeptiv“, ,konkret-gegenständlich“, ,anschaulich“ und „abstrakt-begrifflich“ (Franz und Lange 2014, S. 99), zu erlauben. Bei der Gestaltung von Aufgaben bzw. der Adaption der Lernumgebung sind Zugänge gemäß den Repräsentationsebenen nach Bruner auf symbolischer, ikonischer, enaktiver und kommunikativ-interaktiver Ebene in der Planung des Sachunterrichts zu berücksichtigen (Gebauer und Simon 2012, S. 15 ff.). Es geht um eine Überwindung einer einseitigen Betonung vorrangig schriftbasierter bzw. rein symbolischer Zugangsweisen, da diese als Hindernisse für ein inklusives Lernen im Sachunterricht ausgedeutet werden (etwa Gebauer und Simon 2012; Giest 2015; Kaiser und Teiwes 2003; Seitz 2005).

Die Pluralität dieser Zugangsweisen in Verbindung mit einer Balance von individuellen, wie gemeinschaftlichen und kooperativen Elementen in der gemeinsamen Kommunikation zur Sache, erscheint insbesondere vor dem Hintergrund bedeutsam, 
dass individualisierter bzw. inklusiver Sachunterricht entlang vorgefertigter Werkstätten und Stationsverfahren (Miller und Schroeder 2017) leicht Gefahr läuft, sich lediglich auf eine Differenzierung über niveaugestufte Arbeitsblätter zu realisieren (Spitta 2011, S. 126f.). Insbesondere in der Frage barrierefreier Lehr- und Lernmaterialien wird auch auf den US-amerikanischen Ansatz des universal design for learning im Kontext des Sachunterrichts verwiesen (Schlüter et al. 2016). Die bisherigen deutschsprachigen Umsetzungsbeispiele verbleiben jedoch im Wesentlichen auf der Ebene der zuvor kritisierten, niveaugestuften Arbeitsblattvorlagen, sodass die Potentiale des universal-designs for learning (Meyer et al. 2014) nur begrenzt Anwendung finden. Eine reine Niveaudifferenzierung im Materialangebot würde daher dem formulierten normativen Anspruch an einen inklusiven Sachunterricht, wie dieser etwa durch Seitz (2008) im Konzept eines gemeinsamen Lernens zum Kern der Sache vorgezeichnet ist, nicht gerecht. Um der Unvorherbestimmbarkeit kindlicher Lernweg gerecht zu werden, erscheint es gefordert, gute Lernaufgaben bzw. offene Aufgabenformate zu nutzen (Franz und Lange 2014; Spitta 2011) wie es auch Materialien zum freien Explorieren sowie ästhetischen Erleben und Gestalten (Gebauer und Simon 2012; Schomaker 2008) jenseits (schrift-)sprachbasierter Zugänge (Pech und Rauterberg 2016) bedarf.

\section{Forschungsstand zur Mediennutzung im (inklusiven) Sachunterricht}

Wie entlang des theoretischen Aufrisses skizziert wurde, verortet sich das zugrundliegende Erkenntnisinteresse im Feld fachdidaktischer Forschung zum Sachunterricht (Hartinger 2015) in Schnittmenge zum schulischen Inklusionsdiskurs (Seitz 2018), sodass zentrale Befunde hieraus exemplarisch rezipiert werden. Erste Untersuchungen zum naturwissenschaftlichen Sachunterricht in inklusiven Lerngruppen (Blumberg und Mester 2017; Rott und Marohn 2017) zeigen die Lernwirksamkeit differenzierter, visualisierter, strukturierter und sprachlich vereinfachter Lernangebote mittels entsprechend adaptierter und für die Schülerinnen und Schüler zugänglicher Materialien. Blumberg und Mester (2017, S. 305) nutzen hier in den Experimentalgruppen ihrer Studie eine Kombination von differenzierten sowie auf unterschiedlichen Abstraktionsniveaus und Repräsentationsebenen angelegten Arbeits- und Forscheraufträgen unter Einsatz von elektronischen Lesehilfen, um auch „schwächere“ Kinder der Lerngruppe zu unterstützen. Im Projekt choice ${ }^{2}$ explore (Rott und Marohn 2017) konnten die Kinder durch den Einsatz klar strukturierter, jedoch nicht spezifisch differenzierter Materialien in Leichter Sprache sowie durch Maßnahmen des Scaffoldings in Kleingruppenversuchen Teilchenvorstellungen (weiter-)entwickeln. Im Kontext eines vereinfachten Zugangs zur Schriftsprache in Sachlernprozessen bezieht sich Demir-Walther (2018) in ihrer Interventionsstudie zu förderlichen Wirkungen sprachlich adaptierter Sachtexte auf die Lesekompetenz, das Sachwissen und die emotional-soziale Entwicklung der Kinder. Befunde dieser Studie stehen aber noch aus. An den aufgeführten Interventionsstudien wird demnach die Bedeutung der Lernmaterialien bzw. der darin abgebildeten Lernaufgaben in einem inklusiven Sachunterricht deutlich (auch Schomaker 2015). Demgegenüber scheinen bisherige Lernaufgaben in verfügbaren Sachunterrichtslehrwerken, wie aus 
der Analyse von Kaiser und Albers (2011) hervorgeht, noch kaum Kriterien einer inklusiven Didaktik zu entsprechen. Es fehle vielmehr an Differenzierungsmöglichkeiten und alternativen Zugangsweisen in Lernaufgaben. Spiegler und Ahlgrim (2019) können in ihrer Analyse von Schulbüchern zum Sachunterricht ebenso aufzeigen, dass diese durch stereotype und teils dichotome Darstellungen Differenzkategorien, wie etwa Behinderung, verstärken und nicht kritisch hinterfragen. Diese bisher wenigen, empirischen Schlaglichter auf das Themenfeld Lernmaterialien für einen inklusiven Sachunterricht zeigen einerseits deren Bedeutung auf, andererseits wird auf einen deutlichen Mangel geeigneter Materialien für die Praxis verwiesen.

In einem weiteren Zugriff auf das Forschungsfeld lässt sich an ausgewählten Studien zum Planungshandeln bzw. damit einhergehenden didaktischen Orientierungen von Lehrkräften im Sachunterricht (Giest 2002; Heran-Dörr und Kahlert 2009; Heran-Dörr et al. 2005; Kahlert et al. 2000; Tänzer 2011) ableiten, dass die Verfügbarkeit von Medien und Materialien sowie deren direkte Einsetzbarkeit im Unterricht eine relevante Planungsdeterminante für den Sachunterricht darstellt. Dies hat Tänzer (2011, S. 8) auf Basis ihrer Untersuchung zur Unterrichtsplanung im Sachunterricht als „transferorientierte Materialanalyse“ beschrieben. Demnach lässt sich die hohe Bedeutung, die dem Material als Vermittler zwischen Kind und Sache zugeschrieben wird, erkennen, sodass sich Planung als ,ein materialgeleiteter und materialbedingter Vorgang “ (ebd., S. 10) darstellt. Zu einem ähnlichen Schluss kommen Kahlert et al. (2000) im Ergebnis ihrer Untersuchung zum Planungshandeln von Lehrkräften im Sachunterricht. Ebenso stützen Befunde von Giest (2002) die These, nach der die Auswahl der Ziele und Inhalte des Sachunterrichts wesentlich von vorhandenen Unterrichtsmaterialien beeinflusst wird. Bedenklich müssen hierzu die Befunde von Lange und Wiesemann (2018, S. 131) erscheinen, die in einer ethnografischen Studie zur Gestaltung von Unterrichtsmedien durch die Bildungswirtschaft, herausarbeiten konnten, dass spezifische Selektionsprozess im Vorfeld von Schule determinieren, welche Themen und Inhalte in welcher Weise als vermittelbares Schulwissen gelten können und über Medien Eingang in den Sachunterricht finden. Ein solcher Determinismus durch Lehr- und Lernmedien muss jedoch in deutlichem Widerspruch zu entsprechenden Planungskonzeptionen inklusiven Sachunterricht (etwa Gebauer und Simon 2012; Kaiser und Seitz 2017) bewertet werden, in denen der Kern der Sache (Seitz 2005), als Ausgangspunkt der Planung ko-konstruktiv aus der gemeinsamen Sachbegegnung entstehen soll. Hier muss nach dem Spannungsverhältnis zwischen Materialorientierung und Kindorientierung (Giest 2002; Tänzer 2011) in der Gestaltung des Unterrichts kritisch gefragt werden. So kann insbesondere der verstärkte Fokus auf schriftsprachbasierte Zugangsweisen, im Sinne eines „Arbeitsblattunterrichts “ (Kaiser 2013, S. 18), als wesentliche Barriere für ein inklusives Lernen im Sachunterricht (Gebauer und Simon 2012; Giest 2015; Seitz 2005) betrachtet werden. Inwieweit dies jedoch die gängige Praxis widerspiegelt ist bisher empirisch nicht näher geklärt. Die Frage des Medien- und Materialeinsatzes durch Lehrkräfte in der Praxis des (inklusiven) Sachunterrichts stellt vielmehr ein umfassenderes Forschungsdesiderat dar. 


\section{Forschungsfragen}

Gemäß der skizzierten Relevanz des Medien- und Materialangebotes im Kontext einer inklusiven Sachunterrichtsdidaktik ist übergeordnet die Forschungsfrage nach dem Medien- und Materialeinsatz sowie hierauf wirksamer Einflussfaktoren leitend. Diese lässt sich zu folgenden Teilfragen ausdifferenzieren.

- F1: Welche Medien und Materialien werden von Lehrkräften für den Sachunterricht in Lerngruppen des Gemeinsamen Unterrichts genutzt und welche Möglichkeiten oder Hindernisse sehen sie hierbei?

- F2: Wie begründen Lehrkräfte die Auswahl von Medien und Materialien und was sind handlungsleitende Motive für die getroffenen Auswahlentscheidungen?

Für den vorliegenden Beitrag ${ }^{1}$ erfolgt im Rahmen der spezifischen Forschungsfragen eine Fokussierung auf Merkmale der medialen Angebotsstruktur des Sachunterrichts. Nur insofern sich spezifische und besonders relevante Interdependenzen zur inhaltlich-thematischen sowie methodisch-organisatorischen Ebenen ergeben, sollen diese in begrenztem Maße aufgegriffen werden, um Befunde in ihrem Gesamtkontext zu plausibilisieren.

\section{Forschungsdesign und -methodik}

Methodisch wurde ein zweiphasiges integriertes Mixed-Methods-Design (Creswell 2015) im Kontext einer explorativen Ausrichtung der Untersuchung umgesetzt. In einer ersten Ergebungsphase wurden die Lehrkräfte $(n=80)$ mittels eines teilstandardisierten Fragebogens postalisch befragt. Die Entscheidung für eine Fragebogenerhebung liegt sowohl forschungspragmatisch wie auch gegenstandsspezifisch begründet, da beschreibend zunächst umfassender ein Ist-Stand erhoben werden soll. Die Stichprobenbildung erfolgte über ein geschichtetes Samplingverfahren (Raithel 2008, S. 59) nach Schulamtsbezirken und Schulen, bei dem insgesamt 538 Fragebögen versandt wurden. Durch die Freiwilligkeit der Teilnahme ergibt sich eine Form der Selbstselektionsstichprobe (Döring und Bortz 2016, S. 305 f.), bei der die eingeschränkte Aussagekraft im Kontext der explorativen Zielsetzung zu berücksichtigen ist. Die Rücklaufquote der postalischen Befragung lag mit $15 \%$ im erwartbaren Bereich (ebd., S. 414). Durch Analyse der tatsächlich realisierten Stichprobe kann ein systematischer non-response-Effekt (ebd., S. 296) weitgehend ausgeschlossen werden. Verzerrungen liegen verglichen mit der Zielpopulation vorrangig im höheren Anteil ausgebildeter Sachunterrichtslehrkräfte $(41 \% ; n=33)$ vor, was jedoch durch den spezifischen Interessenfokus der Untersuchung erklärbar ist. Im Fragebogen wurden in einem separaten Fragenblock zunächst über geschlossene Ratingitems

\footnotetext{
1 Die zur Klärung dieser Fragestellungen erhobenen Daten stammen aus einem Dissertationsprojekt zur Erfassung der Sachunterrichtspraxis und handlungsleitender Motive von Lehrkräften im Gemeinsamen Unterricht und an Förderschulen in Nordrhein-Westfalen (etwa Schroeder 2018), worin umfassender Aspekte der inhaltlich-thematischen und methodisch-organisatorischen Ebene der Unterrichtsgestaltung sowie mögliche Einflussfaktoren auf Seiten der Lehrkräfte und Schülerinnen und Schüler erfasst wurden.
} 
Tab. 1 Auszug aus dem Kodierleitfaden zur Hauptkategorie Medien und Materialien

\begin{tabular}{|c|c|c|}
\hline Kategorie & Definition & Ankerbeispiel \\
\hline $\begin{array}{l}\text { 1. Medien und } \\
\text { Materialien }\end{array}$ & $\begin{array}{l}\text { Es werden Aussagen zur Bedeu- } \\
\text { tung von Medien und Materialien im } \\
\text { Sachunterricht getroffen, worunter } \\
\text { Angaben zur Nutzung insgesamt so- } \\
\text { wie Bedingungen und Begründungen } \\
\text { für der Einsatz zusammengefasst } \\
\text { werden }\end{array}$ & - \\
\hline $\begin{array}{l}\text { 1.1. Verfügbarkeit } \\
\text { von Medien und } \\
\text { Materialien }\end{array}$ & $\begin{array}{l}\text { Es werden Aussagen zur Verfügbar- } \\
\text { keit von Medien und Materialien für } \\
\text { den Sachunterricht getroffen, worin } \\
\text { sowohl auf die aktuell verfügbaren } \\
\text { bzw. vorhandenen Materialien ver- } \\
\text { wiesen werden kann wie auch auf } \\
\text { die Notwendigkeit des Verfügbarma- } \\
\text { chens im Sinne der Materialbeschaf- } \\
\text { fung }\end{array}$ & $\begin{array}{l}\text { „Die haben auch schon Informationen } \\
\text { und ich habe auch ... teilweise besorge } \\
\text { eben ich selber dann aus dem Internet } \\
\text { oder so druck ich was aus, bring ich } \\
\text { mit, dass einfach genug Material da } \\
\text { ist." (Interview B7: 26-26) }\end{array}$ \\
\hline $\begin{array}{l}\text { 1.2. Genutzte } \\
\text { Medien und Mate- } \\
\text { rialien }\end{array}$ & $\begin{array}{l}\text { Es wird über die Nutzung bzw. den } \\
\text { Einsatz konkreter Materialien oder } \\
\text { Medien im Sachunterricht berichtet }\end{array}$ & $\begin{array}{l}\text { „Was ich aber mache, ist ... eine Bü- } \\
\text { cherkiste. Also ich mache meistens zu } \\
\text { dem Thema, was ich mache, hole ich } \\
\text { mich vorher in der Bücherei eben eine } \\
\text { Menge Material an Büchern, lasse ich } \\
\text { mir eine Bücherkiste zusammenstellen } \\
\text { und die bring ich dann eben mit, damit } \\
\text { die Kinder immer wieder eine Möglich- } \\
\text { keit haben da rein zu gucken während } \\
\text { unserer Reihe. " (Interview B10: 43-43) }\end{array}$ \\
\hline
\end{tabular}

Häufigkeiten zur Nutzung bestimmter Medienformen im Sachunterricht wie auch verschiedene Einflussfaktoren für den Medieneinsatz im Unterricht erfasst. Weiterhin wurden über offene Antwortformate sowohl Prioritäten in der Mediennutzung wie auch Gründe für die Eignung oder Nicht-Eignung von Unterrichtsmaterialien erfragt. Die vorliegenden quantitativen Daten wurden mittels Methoden der deskriptiven bzw. explorativen Statistik (Döring und Bortz 2016) hinsichtlich vorliegender Verteilungen bzw. Verteilungsunterschiede analysiert. Insbesondere fand im Sinne eines Signifikanztests auf Probe eine interferenzstatistische Prüfung mittels U-Test nach Whitney-Mann hinsichtlich des Merkmales Ausbildungshintergrund der Lehrkraft im Sachunterricht statt. Sofern sich hierbei statistisch bedeutsame Unterschiede im Antwortverhalten nachweisen ließen, wird in der Ergebnisdarstellung darauf Bezug genommen. Die offenen Fragebogenantworten wurden kategorial ausgewertet, um diese in Teilen quantifizieren und deskriptivanalytisch auswerten zu können (Grunenberg und Kuckartz 2013).

Im Anschluss wurden mit einer Teilstichprobe des ursprünglichen Samples $(n=10)$ vertiefende leitfadengestützte Experteninterviews (Bogner et al. 2014) geführt. Im Rahmen einer Triangulationsstrategie (Flick 2011) wurden einzelne Ergebnisse der Fragebogenerhebung als Impulse in die thematische Gesamtstruktur der Interviews integriert (Kruse 2015, S. 213), um Befunde aus der ersten Erhebungsphase kommunikativ zu validieren und Interpretationsräume $\mathrm{zu}$ erweitern. Die Auswertung erfolgte auf Basis einer strukturierenden qualitativen Inhaltsana- 
lyse nach Kuckartz (2014). In einem zirkulären Prozess verschiedener Iterationsschleifen zwischen Forschungsfragen und Material fand eine kombiniert deduktivinduktive Kategoriebildung statt. Das inhaltsanalytische Vorgehen begründet sich sowohl in der spezifischen Einbindung in ein Mixed-Methods-Design (Kuckartz 2014) wie auch dem Interesse am Prozesswissen der Lehrkräfte im Rahmen der Experteninterviews (Bogner et al. 2014). Unter Einbezug der QDA-Software MAXQDA (Kuckartz 2014) wurden in Anlehnung an die Themenblöcke des Leitfadens insgesamt 20 Hauptkategorien herausgearbeitet und das vorhandene Material gemäß dieser Hauptkategorien kodiert, um im Vergleich der kodierten Textstellen weitergehende Subkategorien induktiv zu bestimmen und das Kategoriesystem auszudifferenzieren. Abschließend erfolgte eine Kodierung des gesamten Materials entlang der gebildeten Subkategorien. Für die nachfolgende Ergebnisdarstellung ist hierbei die Hauptkategorie Medien und Materialien von besonderem Interesse. Die Kategoriedefinitionen für diese Hauptkategorie sowie der nachgeordneten ersten Subkategorieebene können exemplarisch der Tab. 1 entnommen werden.

Die Ergebnisse der unterschiedlichen Erhebungsphasen und Datentypen wurden anschließend vor dem Hintergrund der Forschungsfragen trianguliert, um auffällige statistische Befunde plausibilisieren und kontextualisieren zu können sowie insgesamt zu Interpretationen größerer Reichweite zu gelangen (Kelle 2008).

\section{Ergebnisdarstellung}

Zunächst sollen erhobene Befunde zur medialen Angebotsstruktur in Form einer Beschreibung des Ist-Standes sachunterrichtlicher Praxis aus Sicht der befragten Lehrkräfte referiert werden, bevor in einem zweiten Schritt ergänzende Daten zu Begründungszusammenhängen für mediale Entscheidungen der Lehrkräfte erläutert werden. Mittels eines vierstufigen Ratings wurde die Häufigkeit des Medien- und Materialeinsatzes ${ }^{2}$ im Sachunterricht im Rahmen der Fragebogenerhebung erfasst. Entlang der in Tab. 2 dargestellten Häufigkeitsangaben und der aufgetragenen gruppierten Mediane bzw. des Interquartilabstandes lassen sich Unterschiede in der Mediennutzung bestimmen. In ihrer mittleren Tendenz eher häufig finden Arbeitsblätter $(\mathrm{MD}=3,38 ; \mathrm{IQR}=1,20)$, Alltagsmaterialen $(\mathrm{MD}=3,36 ; \mathrm{IQR}=1,16)$ und textbasierte Medien $(\mathrm{MD}=3,31 ; \mathrm{IQR}=1,24)$ Eingang in den Sachunterricht, sodass jeweils über $90 \%$ der befragten Lehrkräfte diese mindestens häufig einsetzen. Demgegenüber werden elektronische Medien, worunter vorrangig der Einsatz von Computern, Tablets oder ähnlichem verstanden wird, gemäß dem ermittelten Median (MD=2,28; $\mathrm{IQR}=1,30)$ tendenziell eher selten von der Mehrzahl der Befragten eingesetzt. In der Prüfung auf signifikante Verteilungsunterschiede im Antwortverhalten mit dem U-Test nach Whitney-Mann ließen sich zwischen den Gruppen der ausgebildeten

\footnotetext{
2 Elektronische Medien (z.B. Textverarbeitung oder Lernspiele am PC, Internet, Filme und MusikCDs), Alltagsmaterialien (z.B. Alltagsgegenstände, Naturmaterialien), pädagogische Lernmaterialien (z.B. Lernspiele, LÜK, Logico, Baukästen, Experimentierboxen), textbasierte Medien (z. B. Sach- oder Kinderbücher, Zeitungsartikel, Sachtexte, Quellentexte), Präsentationsmedien (z. B. Tafelbilder, OHPFolien, Powerpoint-Präsentationen, Karten, Schautafeln), Gestaltungsmedien (z. B. Farben, Papier, Knete, Ton).
} 
Tab. 2 Genutzte Medien und Materialien im Sachunterricht

\begin{tabular}{lccllll}
\hline Item & MD $^{\mathrm{a}}$ & IQR & $\begin{array}{l}n(\%) \\
\text { Sehr sel- } \\
\text { ten/nie }\end{array}$ & Selten & Häufig & Sehr häufig \\
\hline Arbeitsblätter & 3,38 & 1,20 & $1(1,3 \%)$ & $6(7,5 \%)$ & $38(47,5 \%)$ & $35(43,8 \%)$ \\
$\begin{array}{l}\text { Alltagsmaterialien } \\
\text { Textbasierte Me- }\end{array}$ & 3,36 & 1,16 & $0(0,0 \%)$ & $5(6,3 \%)$ & $43(53,8 \%)$ & $32(40,0 \%)$ \\
dien & 3,31 & 1,24 & $0(0,0 \%)$ & $9(11,3 \%)$ & $40(50,0 \%)$ & $31(38,8 \%)$ \\
$\begin{array}{l}\text { Präsentationsmedien } \\
\text { Pädagogische }\end{array}$ & 2,93 & 1,39 & $1(1,3 \%)$ & $23(28,7 \%)$ & $36(45,0 \%)$ & $20(25,0 \%)$ \\
$\begin{array}{l}\text { Lernmaterialien } \\
\text { Gestaltungsmedien }\end{array}$ & 2,79 & 1,26 & $1(1,3 \%)$ & $24(30,0 \%)$ & $44(55,0 \%)$ & $11(13,8 \%)$ \\
$\begin{array}{l}\text { Elektronische } \\
\text { Medien }\end{array}$ & 2,28 & 1,48 & $8(10,0 \%)$ & $25(31,3 \%)$ & $33(41,3 \%)$ & $14(17,5 \%)$ \\
\hline
\end{tabular}

Erhoben wurde über eine vierstufige Skala von $1=$,sehr selten/nie“ bis $4=$,sehr häufig“

${ }^{\mathrm{a}}$ Median gebildet aus gruppierten Daten

Sachunterrichtslehrkräfte und fachfremd unterrichtender Kolleginnen und Kollegen keine statistisch bedeutsamen Unterschiede ermitteln.

Ergänzend wurden die befragten Lehrkräfte im Rahmen eines offenen Antwortformats gebeten, die drei von ihnen im Sachunterricht häufigst genutzten Medien bzw. Materialien in eine Rangreihe zu bringen. Die kategoriale Auswertung erfolgt in Analogie zu den zuvor eingesetzten Antwortkategorien. Nachfolgend wurde für die statistische Auswertung in umgekehrter Weise zu ihrem Rangplatz jeweils ein Wert von 3 bis 0 zugewiesen. Hieraus ergibt sich, wie in Abb. 1 dargestellt, aufsteigend eine Rangreihe, in der sich die Priorisierungen der Medien bzw. Materialien für den Sachunterricht widerspiegeln. Auch hier führen Arbeitsblätter $(\mathrm{MD}=1,80$; IQR = 2,22) mit deutlichem Abstand die gebildete Rangreihe an. Ebenfalls korrespondiert mit dem Antwortverhalten zu den zuvor abgefragten Häufigkeiten der Mediennutzung, finden sich textbasierte Medien $(\mathrm{MD}=1,00 ; \mathrm{IQR}=1,86)$ sowie Alltagsmaterialien $(\mathrm{MD}=0,83 ; \mathrm{IQR}=2,12)$ auf den nachfolgenden Rängen. Erneut werden elektronische Medien $\left(\mathrm{MD}=0,14 ; \mathrm{IQR}=\mathrm{n} . \mathrm{def}^{3}{ }^{3}\right)$ bzw. Gestaltungsmedien $\left(\mathrm{MD}=0,16 ; \mathrm{IQR}=\mathrm{n}\right.$. def. $\left.{ }^{1}\right)$ nur von sehr wenigen Lehrkräften als prioritär für den Sachunterricht bestimmt. Darüber hinaus nutzen lediglich $18 \%$ der Befragten ein Sachunterrichtslehrwerk. Dies wurde separat erfragt.

Im Ergebnis der quantitativen Auswertung lässt sich eine eindeutige Präferenz für die Nutzung von Arbeitsblättern und textbasierten Medien, noch deutlich vor allen anderen Medienformen, erkennen. Innerhalb der gebildeten Rangreihen wird die hervorgehobene Bedeutung von Arbeitsblättern als zentralem Medium im Sachunterricht sichtbar, obwohl auch die meisten anderen Medienformen regelmäßige Nutzung erfahren. Allerdings erlauben die dargestellten quantitativen Daten an dieser Stelle keine weitergehenden Aussagen darüber, welche Medien oder Materialien spezifisch eingesetzt bzw. wie diese in den Sachunterricht genauer eingebunden

\footnotetext{
3 Der Interquartilabstand als Maß für Streuung der Wert ist an dieser Stelle nicht definierbar, da sich die unteren Quartile nicht hinreichend differenzieren lassen.
} 


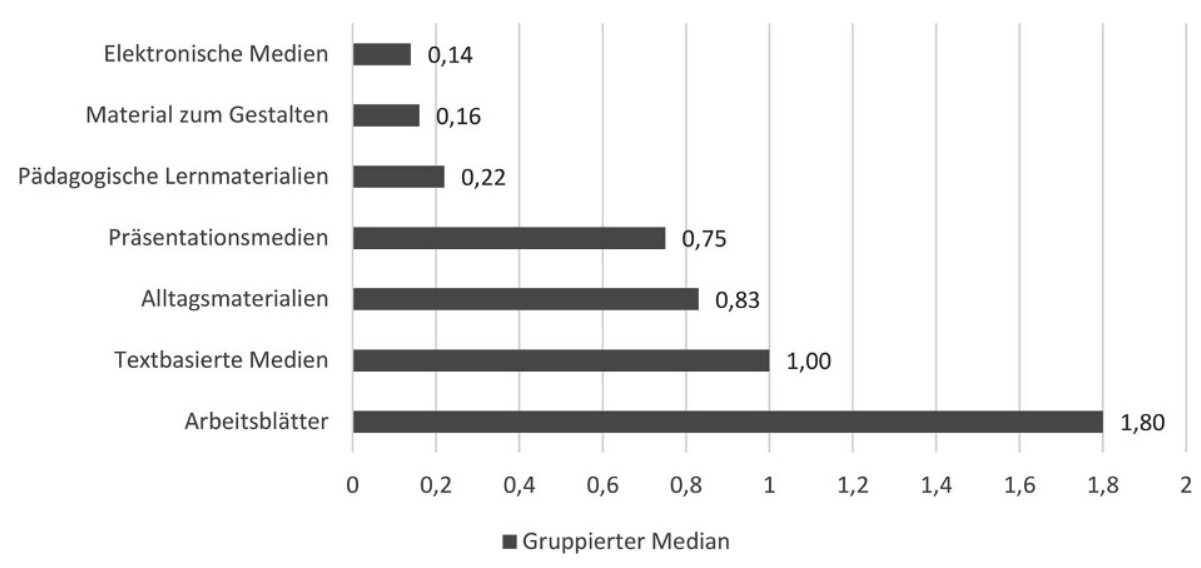

Abb. 1 Priorisierte Medien und Materialien im Sachunterricht

werden. Ergänzende Hinweise ergeben sich hierzu aus der inhaltsanalytischen Auswertung der Experteninterviews.

Entsprechende Aussagen im Interviewmaterial wurden der Oberkategorie Medien und Materialien zugeordnet, auf die sich jedoch insgesamt nur 60 Codings beziehen. Dies ist dahingehend einzuordnen, dass auf die parallelen Oberkategorien Inhaltsauswahl 480 Codings bzw. Methodische Ebene 543 Codings entfallen, sodass die mediale Angebotsstruktur in den Beschreibungen vordergründig eine untergeordnete Rolle zu spielen scheint. Zur Subkategorie Genutzte Medien und Materialien lassen sich 28 Codings ermitteln, aus denen Angaben über den konkreten Material- und Medieneinsatz hervorgehen. So wird von vier interviewten Lehrkräften die Nutzung von Büchern (4) genannt oder von jeweils drei Lehrkräften auf den Einsatz von Kopiervorlagen (6) oder Filmen (3) verwiesen. Ebenfalls werden Experimentierboxen (3), Alltagsmaterialien (2), Computer (2) oder die Tafel (2) beschrieben. Bedenkenswert ist, dass im Vergleich zur Dominanz des Arbeitsblattes in den quantitativen Ergebnissen, dies eher peripher in den Interviewaussagen (Kategorie: Kopiervorlagen) erscheint. Dabei wird der Nutzen solcher Kopiervorlagen für die eigene Praxis von den interviewten Lehrkräften sehr unterschiedlich bewertet. Einerseits werden die Einsatzmöglichkeiten als sehr eingeschränkt erlebt, indem etwa eine Lehrkraft ausführt: „Und da kann ich diese Werkstätten vom Verlag an der Ruhr zum Beispiel oft nicht gebrauchen, ne" (Interview B4: 14-14). Andererseits wird der deutliche Nutzen für die eigene Sachunterrichtspraxis betont: „und dann waren also ganz viele Sachen schon so fertig und das fand ich einfach ganz toll die zu probieren" (Interview B5: 75-75). Ähnlich divergierende Einschätzungen finden sich auch gegenüber vorgefertigten Experimentierboxen (3), indem die didaktisierten Materialien sowohl als hilfreiche Unterstützung erlebt wie auch als stark einschränkend hinsichtlich eines freieren Explorierens der Schülerinnen und Schüler erfahren werden. Relevant für eine nachgehende Interpretation ist, dass die besondere Nützlichkeit vorgefertigter Kopiervorlagen bzw. Experimentierboxen jeweils von fachfremd unterrichtenden Lehrkräften artikuliert wird, wohingegen der kritische Blick auf deren Einsatzmöglichkeiten von ausgebildeten Sachunterrichtslehrkräften stammt. 
Tab. 3 Gestaltung von Unterrichtsmaterialien für die Lerngruppe

\begin{tabular}{lccllll}
\hline Item & MD $^{\mathrm{a}}$ & $\mathrm{IQR}$ & $\begin{array}{l}n(\%) \\
\text { Sehr sel- } \\
\text { ten/nie }\end{array}$ & Selten & Häufig & Sehr häufig \\
\hline $\begin{array}{l}\text { Selbstgestaltung } \\
\text { von Materialien } \\
(n=80)\end{array}$ & 3,24 & 1,28 & $0(0,0 \%)$ & $12(15,0 \%)$ & $40(50,0 \%)$ & $28(35,0 \%)$ \\
$\begin{array}{l}\text { Anpassung von } \\
\text { Materialien an }\end{array}$ & 3,26 & 1,18 & $0(0,0 \%)$ & $6(7,5 \%)$ & $48(60,0 \%)$ & $25(31,3 \%)$ \\
$\begin{array}{l}\text { Lerngruppe } \\
(n=79)\end{array}$ & & & & & & \\
\hline
\end{tabular}

Erhoben wurde über eine vierstufige Skala von $1=$,sehr selten/nie“ bis $4=$,sehr häufig“

${ }^{\mathrm{a}}$ Median gebildet aus gruppierten Daten

Hiermit wird die Frage nach der Passung der Materialien zur jeweiligen Lerngruppe bzw. zum eigenen Unterricht sowie einer ggf. notwendigen Adaptionsleistung durch die Lehrkraft aufgeworfen. Im Rahmen der Fragebogenerhebung wurde erfasst, wie häufig die Lehrkräfte Unterrichtsmaterialien für ihre Lerngruppe selbst erstellen oder wie oft eine Anpassung vorhandener Materialien notwendig ist. Es zeigt sich (siehe auch Tab. 3), dass sowohl die Anpassung von Materialien ( $\mathrm{MD}=3,26$; $\mathrm{IQR}=1,18)$ wie auch die eigenständige Gestaltung $(\mathrm{MD}=3,24 ; \mathrm{IQR}=1,18)$ von der überwiegenden Mehrheit der befragten Lehrkräfte als mindestens häufige Tätigkeit in der Vorbereitung des Sachunterrichts wahrgenommen wird. Damit zusammenhängend können $64 \%(n=50)$ der befragten Lehrkräfte der Aussage zustimmen, dass verfügbare Sachunterrichtsmaterialien für ihre Lerngruppe nur bedingt geeignet erscheinen. Um Gründe für eine eingeschränkte Eignung von Materialien für die eigene Lerngruppe zu erfassen, wurden diese ergänzend über ein offenes Frageitem eruiert. Aus der kategorialen Auswertung geht hervor, dass zentrale Gründe in fehlenden Differenzierungsmöglichkeiten (19), fehlender Strukturierung (13), einem zu hohen Anspruchsniveau (9) oder einem zu hohen Textanteil (8) gesehen werden. Gleichzeitig spielt die Verfügbarkeit sowie der Kostenaufwand von Materialien eine relevante Rolle bei der Planung des Sachunterrichts $(\mathrm{MD}=3,13 ; \mathrm{IQR}=1,28)$. So trifft dies für $56 \%$ eher zu $(n=45)$ bzw. für $26 \%$ voll zu $(n=21)$.

Aus der Frage nach Motiven für die Themen- und Inhaltsauswahl geht hervor, dass nur für 16\% $(n=13)$ das vorhandene Materialangebot leitend ist, sich lediglich $9 \%(n=7)$ durch Verlagsanregungen prioritär Anregungen holten und für $9 \%$ $(n=7)$ der Aufbau des Lehrwerkes als Vorgabe genutzt wird. Andere, inhaltlich-intentionale Planungsmotive werden demnach erheblich höher gewichtet. Signifikante Verteilungsunterschiede zwischen ausgebildeten und fachfremden Lehrkräften ließen sich dabei nicht feststellen.

Konträr hierzu wird die Bedeutung von Medien und Materialien für die Planung des Sachunterrichts in den Experteninterviews deutlich erkennbar. Zentrale Begründungsmotive für die Inhaltsauswahl sind pragmatische Argumente der Verfügbarkeit von Materialien (29) oder der notwendige Vorbereitungsaufwand (5). Bestimmte Inhalte werden demnach thematisiert, weil es „viele Sachen drüber gibt“ (Interview B5: 61-61) oder „Magnetismus ist auch ein schönes Thema. Gibt es auch sehr viel drüber“ (Interview B4: 44-44). Gute bzw. leicht zu unterrichtende Themen sind 
demnach solche, zu denen viele Materialien verfügbar sind. Ebenso erfolgt die $M e-$ thodenauswahl nicht unwesentlich mit Blick auf die Verfügbarkeit von Medien und Materialien (17). Expliziert wird hierbei etwa der spezifische Aufwand, der bereits in der Fragebogenerhebung als Kriterium bestimmt wurde, benannt: „Ich muss es erstellen und da ist dann die Frage, ob ich als Lehrer dann immer bereit bin dafür Zeit aufzubringen. Deshalb denke ich, dass die Häufigkeit der Methoden auch ganz. klar von so was abhängt" (Interview B1: 46-46). Im Vergleich zu Kind orientierten Begründungsmustern, wie etwa Voraussetzungen und Erfahrungen der Schülerinnen und Schüler (82) oder Interessenorientierung (19) spielt das Motiv der Materialorientierung zwar eine nachrangige Rolle, muss aber als weitere, zentrale Begründungsfigur für didaktische Entscheidungen im Sachunterricht gelten. Korrespondierend wird in den Experteninterviews die Materialbeschaffung (9) als eine wesentliche Aufgabe in der Vorbereitung des Sachunterrichts wahrgenommen. „Informationen und ich habe auch ... teilweise besorge eben ich selber dann aus dem Internet oder so drucke ich was aus, bring ich mit, dass einfach genug Material da ist" (Interview B7: 26-26). So wird durch die Lehrkraft B7 die Bedeutung des Internets als eine Quelle für Materialien und Informationen in der Vorbereitung des Sachunterrichts benannt. 9 von 10 der interviewten Lehrkräfte beschreiben an ihren Schulen für den Sachunterricht übergreifende Ausstattungsprobleme (20). Diese Schwierigkeiten resultierten sowohl aus dem Nicht-Vorhandensein wie auch der Nicht-Eignung von Materialien, wie dies etwa die Befragte B1 ausführt: „Also wir haben kaum Materialien. Die Materialien, die wir haben sind nicht vollständig “ (Interview B1: 81-81). Insgesamt werden die Rahmenbedingungen für den Sachunterricht hinsichtlich der medialen Ausstattung als einschränkender Faktor erlebt: „Deshalb (b lacht) unterstützen tut mich hier leider sehr wenig " (Interview B1: 81-81).

\section{Zusammenfassende Ergebnisinterpretation}

Mit Blick auf die formulierte Forschungsfrage $F 1$ scheinen die befragten Lehrkräfte grundsätzlich ein vielfältiges Medien- und Materialangebot in ihrem Sachunterricht bereitzustellen. So werden die meisten Medienformen, die in der Erhebung abgefragt wurden, von einem Großteil der Lehrkräfte mindestens häufig im Sachunterricht eingesetzt. Dies trifft insbesondere auf Arbeitsblätter, Alltagsmaterialien und textbasierte Medien zu, die das Kernelement der mediale Angebotsstruktur ausmachen. Neben einem vorrangig schriftsprachbasierten Zugang über Arbeitsblätter und textbasierte Medien werden mit dem Einsatz von Alltagsmaterialien auch enaktive Repräsentationsformen bereitgestellt, was als Anspruch an einen inklusiven Sachunterricht gelten muss (Gebauer und Simon 2012; Kaiser und Seitz 2017). Gleichzeitig erscheint das Arbeitsblatt für die sachunterrichtliche Praxis von vorrangiger Relevanz. Der Sachunterricht wird in nicht unwesentlichen Teilen als der von Kaiser (2013, S. 18) kritisierte „Arbeitsblattunterricht“ gestaltet, obwohl ebenso textbasierte Medien, Präsentationsmedien und Alltagmaterialien häufig in Ergänzung hierzu eingesetzt werden. Ob dies in Summe damit die pluralen Zugangs- und Aneignungswege ermöglicht, wie dies als Prämisse in vorliegenden Konzeptionen inklusiven Sachunterrichts betont wird, muss jedoch fraglich bleiben. Medien und 
Materialien zum ästhetischen Gestalten, aber auch pädagogische Lernmaterialien werden zumindest kaum als prioritär für die mediale Angebotsstruktur bestimmt.

Korrespondierend mit den zuvor referierten Untersuchungen (etwa Giest 2002; Kahlert et al. 2000; Tänzer 2011) wird insgesamt die hohe Bedeutung von Medien und Materialien für die Planung des Sachunterrichts in den Experteninterviews erkennbar. In Beantwortung der zweiten Forschungsfrage (F2) wird deutlich, dass der Planungsfokus entgegen den Forderungen aus dem Fachdiskurs nicht in dem Maß auf der Perspektivität der Kinder (Kaiser und Seitz 2017; Seitz 2005) liegt, sondern in Teilen von der Verfügbarkeit bzw. Struktur des Materials determiniert wird. Hierbei weisen die erhobenen Einschätzungen der Lehrkräfte zur Eignung und Verfügbarkeit von Materialien diese ebenso als spezifische Problemstellen aus. Gründe liegen in fehlenden Differenzierungsmöglichkeiten oder unzureichender Strukturierung, was mit empirisch belegten Kriterien zur Lernwirksamkeit zusammenfällt (Blumberg und Mester 2017; Rott und Marohn 2017). Gleichzeitig lässt sich ein Dilemma zwischen einem Wunsch nach Unterstützung durch weitgehend vorgefertigte und stärker didaktisierte Materialien einerseits und einer zwangsläufig fehlenden exakten Passung für die Bedürfnisse der jeweils eigenen Lerngruppe erkennen. Dieses wird von ausgebildeten Sachunterrichtslehrkräften deutlicher wahrgenommen, als dies für fachfremd unterrichtende Lehrkräfte gilt.

Hinsichtlich der ebenfalls erfassten handlungsleitenden Motive zeigen sich deutliche Ambivalenzen im Vergleich von Fragebogen- und Interviewauswertung. Erhalten in der Fragebogenuntersuchung Items, die eine Materialorientierung als Planungsmotiv erfassen, nur geringe mittlere Zustimmungswerte, so lässt sich aus der Analyse des Interviewmaterials ein Planungsmotiv der Materialorientierung deutlich herausarbeiten. Übereinstimmungen ergeben sich in der Triangulation der Befunde hingegen in wahrgenommenen Hindernissen für eine inklusive Sachunterrichtspraxis durch ein fehlendes oder ungeeignetes Material- und Medienangebot. Die Materialbeschaffung tritt als wesentlicher Teil der Unterrichtvorbereitung in den Vordergrund sachunterrichtlicher Planungs- und Vorbereitungstätigkeit.

\section{Ausblick}

Eine mögliche Aufgabe für sachunterrichtsdidaktische Forschung, die sich schlussfolgernd aus den Ergebnissen ergibt, könnte die verstärkte Entwicklung geeigneter Medien und Materialien, etwa entlang der Prinzipien des ,, universal design for learning " (Meyer et al. 2014; Schlüter et al. 2016) für einen inklusiven Sachunterricht sein, um so Lehrkräfte in ihrer Praxis zu unterstützen. Hier gilt es insbesondere die sichtbare Grundstruktur eines „Arbeitsblattunterrichts “ aufzubrechen, da dieser kaum geeignet erscheinen muss, die zuvor formulierten Ansprüche einer inklusiven Sachunterrichtsdidaktik von Personalität, Sozialität und Komplexität (Kaiser und Seitz 2017, S. 12) einzulösen. Wenn die in den verfügbaren Materialien inhärent angelegte didaktische Struktur an die Stelle reflektierter Entscheidungen zwischen Kind- und Sachorientierung tritt, muss dies als deutliche Problemstelle für eine inklusiven Sachunterricht gelten. Statt vielfältiger Zugangsweisen und einem Lernen auf verschiedenen Repräsentationsebene dominieren im Ergebnis dieser Planungs- 
prozesse Arbeitsblätter die Praxis des Sachunterrichts. Ambivalent hierzu verweisen die Befunde darauf, dass Sach- und Kindorientierung vorangestellte Motive sachunterrichtlicher Planungen sind, jedoch das verfügbare Medien- und Materialangebot es fraglich erscheinen lässt, ob damit konnotierte Zielsetzungen konsequent umgesetzt werden können. Barrierefreiheit im Sinne eines ,, universal design for learning “ bedeutet dann nicht, wie dies erste deutschsprachige Rezeptionen (Schlüter et al. 2016) nahelegen, allein eine Reduktion auf vielfältige Wege der Darbietung durch differenzierte Medien, sondern betrifft entlang der drei Leitprinzipien des Ansatzes (etwa Meyer et al. 2014) wesentlich die Ziel- und Inhaltsebene des Fachunterrichts und die Beteiligung aller Kinder. Barrierefreie Lehr- und Lernmaterialien sind demnach ein Baustein eines inklusiven Sachunterrichts, der jedoch nachgängig zur Frage steht, wie subjektiv bedeutsame Sachbegegnung für alle Kinder ermöglicht werden kann. Ausgangspunkt des „Warum “ des Lernens (,Engagement") liegt damit in den Interessen und Sichtweisen der Kinder selbst, muss aber in dem „Was“ des Lernens (,Representation") auch von der Sache gedacht werden, um wiederum im „Wie“ des Lernens (,Action \& Expressions") der Vielfalt der Lernvoraussetzungen und -weg zu entsprechen. In der Frage individuell wie gesellschaftlich anschlussfähiger Sachbildung kann dies die Notwendigkeit eines erweiterten Theorie-Praxis-Diskurses über Ziele, Themen und Inhalte des Sachunterrichts im Verhältnis von Kind und Sache (vgl. Pech et al. 2018) implizieren.

Funding Open Access funding provided by Projekt DEAL.

Open Access Dieser Artikel wird unter der Creative Commons Namensnennung 4.0 International Lizenz veröffentlicht, welche die Nutzung, Vervielfältigung, Bearbeitung, Verbreitung und Wiedergabe in jeglichem Medium und Format erlaubt, sofern Sie den/die ursprünglichen Autor(en) und die Quelle ordnungsgemäß nennen, einen Link zur Creative Commons Lizenz beifügen und angeben, ob Änderungen vorgenommen wurden.

Die in diesem Artikel enthaltenen Bilder und sonstiges Drittmaterial unterliegen ebenfalls der genannten Creative Commons Lizenz, sofern sich aus der Abbildungslegende nichts anderes ergibt. Sofern das betreffende Material nicht unter der genannten Creative Commons Lizenz steht und die betreffende Handlung nicht nach gesetzlichen Vorschriften erlaubt ist, ist für die oben aufgeführten Weiterverwendungen des Materials die Einwilligung des jeweiligen Rechteinhabers einzuholen.

Weitere Details zur Lizenz entnehmen Sie bitte der Lizenzinformation auf http://creativecommons.org/ licenses/by/4.0/deed.de.

\section{Literatur}

Blumberg, E., \& Mester, T. (2017). Potentielle Gelingensbedingungen im naturwissenschaftlichen Sachunterricht der Grundschule - auf dem Weg zu empirischen Evidenzen. In F. Hellmich \& E. Blumberg (Hrsg.), Inklusiver Unterricht in der Grundschule (S. 294-312). Stuttgart: Kohlhammer.

Bogner, A., Littig, B., \& Menz, W. (2014). Interviews mit Experten. Eine praxisorientierte Einführung. Wiesbaden: Springer VS.

Creswell, J.W. (2015). A concise introduction to mixed methods research. Thousand Oaks: SAGE.

Demir-Walther, M. (2018). Die Entwicklung eines Unterrichtskonzeptes zur Förderung von Sachlernen und Lesekompetenz aus inklusionsdidaktischer Sicht - ein Unterricht für Kinder mit Down-Syndrom. In D. Pech, C. Schomaker \& T. Simon (Hrsg.), Sachunterrichtsdidaktik \& Inklusion. Ein Beitrag zur Entwicklung (S. 39-44). Baltmansweiler: Schneider Verlag Hohengehren.

Döring, N., \& Bortz, J. (2016). Forschungsmethoden und Evaluation in den Sozial- und Humanwissenschaften (5. Aufl.). Berlin; Heidelberg: Springer.

Flick, U. (2011). Triangulation. Eine Einführung. Wiesbaden: VS. 
Franz, E.-K., \& Lange, B. (2014). Lernaufgaben im Rahmen inklusiver Didaktik. In E.-K. Franz, S. Trumpa \& I. Esslinger-Hinz (Hrsg.), Inklusion: Eine Herausforderung für die Grundschulpädagogik (S. 97-107). Baltmannsweiler: Schneider Verlag Hohengehren.

Gebauer, M., \& Simon, T. (2012). Inklusiver Sachunterricht konkret. Chancen, Grenzen, Perspektiven. http://www.widerstreit-sachunterricht.de. Zugegriffen: 13. Juni 2019.

Giest, H. (2002). Entwicklungsfaktor Unterricht. Empirische Untersuchungen zum Verhältnis von Unterricht und Entwicklung in der Grundschule. Dargestellt am Beispiel des Heimatkunde- und Sachkundeunterrichts. Landau: Verlag Empirische Pädagogik.

Giest, H. (2015). Diagnostik und Inklusion im Sachunterricht. In H. Schäfer \& C. Rittmeyer (Hrsg.), Handbuch Inklusive Diagnostik (S. 214-229). Weinheim, Basel: Beltz.

Grunenberg, H., \& Kuckartz, U. (2013). Deskriptive Statistik in der qualiativen Sozialforschung. In B. Friebertshäuser, A. Langer \& A. Prengel (Hrsg.), Handbuch Qualiative Forschungsmethoden in der Erziehungswissenschaft (4. Aufl. S. 487-500). Weinheim und Basel: Beltz Juventa.

Hartinger, A. (2015). Empirische Zugänge. In J. Kahlert, M. Fölling-Albers, A. Hartinger, S. Miller \& S. Wittkowske (Hrsg.), Handbuch Didaktik des Sachunterrichts (S. 47-51). Bad Heilbrunn: Klinkhardt.

Heckt, D. H. (2005). Medien, Arbeitsmittel, Materialien. In W. Einsiedler, M. Götz, H. Hacker, J. Kahlert, R. W. Keck \& U. Sandfuchs (Hrsg.), Handbuch Grundschulpädagogik und Grundschuldidaktik (2. Aufl. S. 449-454). Bad Heilbrunn: Klinkhardt.

Heran-Dörr, E., \& Kahlert, J. (2009). Welche Medien nutzen Sachunterrichtslehrkräfte bei der Vorbereitung auf naturwissenschaftlichen Sachunterricht? In R. Lauterbach, H. Giest \& B. Marquardt-Mau (Hrsg.), Lernen und kindliche Entwicklung. Elementarbildung und Sachunterricht (S. 157-164). Bad Heilburnn: Klinkhardt.

Heran-Dörr, E., Kahlert, J., \& Wiesner, H. (2005). Internetunterstütze Lehrerfortbildung zur Förderung von fachdidaktischer Expertise im naturwissenschaftlichen Sachunterricht. In D. Cech \& H. Giest (Hrsg.), Sachunterricht in Praxis und Forschung (S. 139-146). Bad Heilbrunn: Klinkhardt.

Kahlert, J. (2016). Der Sachunterricht und seine Didaktik (4. Aufl.). Bad Heilbrunn: Klinkhardt.

Kahlert, J., Hedtke, R., \& Schwier, V. (2000). Wenn Lehrer wüssten, was Lehrer wissen. Beschaffung von Informationen für den Unterricht. In O. Jaumann-Graumann \& W. Köhnlein (Hrsg.), Lehrerprofessionalität - Lehrerprofessionalisierung (S. 349-358). Bad Heilbrunn: Klinkhardt.

Kaiser, A. (2013). Kommunkativer Sachunterricht. In A. Becher, S. Miller, I. Oldenburg, D. Pech \& C. Schomaker (Hrsg.), Kommunikativer Sachunterricht. Facetten der Entwicklung (S. 13-25). Baltmannsweiler: Schneider Verlag Hohrengehren.

Kaiser, A., \& Albers, S. (2011). Inklusion durch Lernaufgaben in Sachunterrichtsbüchern - ein Widerspruch? In H. Giest, A. Kaiser \& C. Schomaker (Hrsg.), Sachunterricht-auf dem Weg zur Inklusion (S. 79-88). Bad Heilbrunn: Klinkhardt.

Kaiser, A., \& Seitz, S. (2017). Inklusiver Sachunterricht. Theorie und Praxis. Baltmannsweiler: Schneider Verlag Hohengehren.

Kaiser, A., \& Teiwes, K. (2003). Handelndes Lernen im Sachunterricht auch für Kinder mit besonderem Förderbedarf. In D. Cech \& H.-J. Schwier (Hrsg.), Lernwege und Aneignungsformen im Sachunterricht (S. 173-186). Bad Heilbrunn: Klinkhardt.

Kelle, U. (2008). Die Integration qualitativer und quantiativer Methoden in der empirischen Sozialforschung. Theoretische und methodologische Konzepte (2. Aufl.). Wiesbaden: VS.

Klafki, W. (2007). Neue Studien zur Bildungstheorie und Didaktik: zeitgemäße Allgemeinbildung und kritisch-konstruktive Didaktik (6. Aufl.). Weinheim; Basel: Beltz.

Köhnlein, W. (2010). Planung von Sachunterricht aus dem didaktischen Primat der Sache. In S. Tänzer \& R. Lauterbach (Hrsg.), Sachunterricht begründet planen. Bedingungen, Entscheidungen, Modelle (S. 165-178). Bad Heilbrunn: Klinkhardt.

Kruse, J. (2015). Qualitative Interviewforschung. Ein integrativer Ansatz. (2. Aufl.). Weinheim; Basel: Beltz Juventa.

Kuckartz, U. (2014). Qualitative Inhaltsanalyse. Methoden, Praxis, Computerunterstützung (2. Aufl.). Weinheim, Basel: Beltz Juventa.

Lange, J., \& Wiesemann, J. (2018). Schulische Experimente als Produkt der Bildungswirtschaft. In U. Franz, H. Giest, A. Hartinger, A. Heinrich-Dönges \& B. Reinhoffer (Hrsg.), Handeln im Sachunterricht (S. 125-132). Bad Heilbrunn: Klinkhardt.

Meyer, A., Rose, D.H., \& Gordon, D. (2014). Universal design for learning. Wakefield: Cast.

Miller, S. \& Schroeder, R. (2017). Werkstatt und Stationenlernen. In D. von Reeken (Hrsg.), Handbuch Methoden im Sachunterricht (3, Aufl. S. 283-292). Baltmannsweiler: Schneider Verlag. 
Mitzlaff, H. (2016). Medien inklusive - inklusive Mediendidaktik. In M. Peschel (Hrsg.), Mediales Lernen. Beispiele für eine inklusive Mediendidaktik (S. 17-34). Baltmannsweiler: Schneider Verlag Hohengehren.

Pech, D., \& Rauterberg, M. (2016). Wozu Didaktik? Ein Beitrag zum Verhältnis von Sachunterrichtsdidaktik und Inklusion. In O. Musenberg \& J. Riegert (Hrsg.), Didaktik und Differenz (S. 134-147). Bad Heilbrunn: Klinkhardt.

Pech, D., Schomaker, C., \& Simon, T. (2017). Inklusive Fachdidakitk Sachunterricht. In K. Ziemen (Hrsg.), Lexikon Inklusion (S. 124-125). Göttingen: Vandenhoek \& Ruprecht.

Pech, D., Schomaker, C., \& Simon, T. (2018). Inklusion sachunterrichts-didaktisch gedacht. In D. Pech, C. Schomaker \& T. Simon (Hrsg.), Sachunterricht \& Inklusion: Ein Beitrag zur Entwicklung (S. 10-25). Baltmannsweiler: Schneider Verlag Hohengehren.

Peschel, M. (2016). Mediales Lernen - Eine Modellierung als Einleitung. In M. Peschel (Hrsg.), Mediales Lernen. Beispiele für eine inklusive Mediendidaktik (S. 7-16). Baltmannsweiler: Schneider Verlag Hohengehren.

Raithel, J. (2008). Quantitative Forschung. Ein Praxiskurs (2. Aufl.). Wiesbaden: VS.

Rott, L., \& Marohn, A. (2017). Choice2explore - eine an Schülervorstellungen orientierte Unterrichtskonzeption für den inklusiven Sachunterricht. GDSU-Journal, 2017(7), 105-116.

Schlüter, A.-K., Melle, I., \& Wember, F.B. (2016). Unterrichtsgestaltung in Klassen des Gemeisamen Lernens. Universal Design of Learning. Sonderpädagogische Förderung heute, 2016(3), 270-285.

Schomaker, C. (2008). Ästhetische Bildung im Sachunterricht. Zur kritisch-reflexiven Dimension ästhetischen Lernens. Baltmannsweiler: Schneider Verlag Hohengehren.

Schomaker, C. (2013). Inklusiver Sachunterricht - Umsetzungsformen und Wege in der Praxis. In E. Gläser \& G. Schönknecht (Hrsg.), Sachunterricht in der Grundschule entwickeln - gestalten - reflektieren (S. 295-305). Frankfurt am Main: Grundschulverband.

Schomaker, C. (2015). Individuell und gemeinsam an Sachen lernen. Zur Gestaltung von Aufgaben in einem lernförderlichen Sachunterricht. In I. B. Behrensen, E. Gläser \& C. Solzbacher (Hrsg.), Fachdidakitk und individuelle Förderung in der Grundschule. Perspekiven auf Unterricht in heterogenen Lerngruppen (S. 109-116). Baltmannsweiler: Schneider Verlag Hohengehren.

Schroeder, R. (2018). Inklusiver Sachunterricht - alles neu? Befunde zur bestehenden Sachunterrichtspraxis im Gemeinsamen Unterricht der Grundschulen. In D. Pech, C. Schomaker \& T. Simon (Hrsg.), Sachunterrichtsdidaktik \& Inklusion. Ein Beitrag zur Entwicklung (S. 54-68). Baltmannsweiler: Schneider Verlag Hohengehren.

Seitz, S. (2005). Zeit für inklusiven Sachunterricht. Baltmannsweiler: Schneider Verlag Hohengehren.

Seitz, S. (2008). Zu einer inklusiven Didaktik des Sachunterrichts. In A. Kaiser \& D. Pech (Hrsg.), Integrative Dimenionen für den Sachunterricht. Neuere Zugangsweisen (2. Aufl. S. 169-180). Baltmannsweiler: Schneider Verlag Hohengehren.

Seitz, S. (2018). Forschung zu inklusivem Sachunterricht - Bestandsaufnahme und Perspektiven. In D. Pech, C. Schomaker \& T. Simon (Hrsg.), Sachunterricht \& Inklusion. Ein Beitrag zur Entwicklung (S. 96-111). Baltmannsweiler: Schneider Verlag Hohengeheren.

Spiegler, J., \& Ahlgrim, T. (2019). „Beratet, wie ihr in der Klasse mit Lioba zusammen lernen und leben würdet.“ Darstellung von ,Behinderung“ in Schulbüchern des Sachunterrichts. In D. Pech, C. Schomaker \& T. Simon (Hrsg.), Inklusion im Sachunterricht. Perspektiven der Forschung (S. 102-112). Bad Heilbrunn: Klinkhardt.

Spitta, P. (2011). „Wie kann man ohne Arbeitsblätter differenzieren?“ - Zum Stellenwert von Inklusion in der Lehrer/innenausbildung. In H. Giest, A. Kaiser \& C. Schomaker (Hrsg.), Sachunterricht - auf dem Weg zur Inklusion (S. 123-131). Bad Heilbrunn: Klinkhardt.

Tänzer, S., \& Lauterbach, R. (Hrsg.). (2010). Sachunterricht begründet planen. Bedingungen, Entscheidungen, Modelle. Bad Heilbrunn: Klinkhardt.

Tänzer, S. (2011). Sachunterrichtsplanung aus Sicht von Lehramtsanwärterinnen. http://www.widerstreitsachunterricht.de. Zugegriffen: 13. Juni 2019.

Tulodziecki, G. (2011). Medien und Lernmittel im Grundschulunterricht. In W. Einsiedler, M. Götz, A. Hartinger, F. Heinzel, J. Kahlert \& U. Sandfuchs (Hrsg.), Handbuch Grundschulpädagogik und Grundschuldidaktik (3. Aufl. S. 402-409). Bad Heilbrunn: Klinkhardt.

Unglaube, H. (2015). Arbeitsmittel. In J. Kahlert, M. Fölling-Albers, M. Götz, A. Hartinger, S. Miller \& S. Wittkowske (Hrsg.), Handbuch Didaktik des Sachunterrichts (2. Aufl. S. 491-495). Bad Heilbrunn: Klinkhardt.

Wocken, H. (2013). Das Haus der inklusiven Schule. Baustellen - Baupläne - Bausteine (4. Aufl.). Hamburg: Feldhaus. 